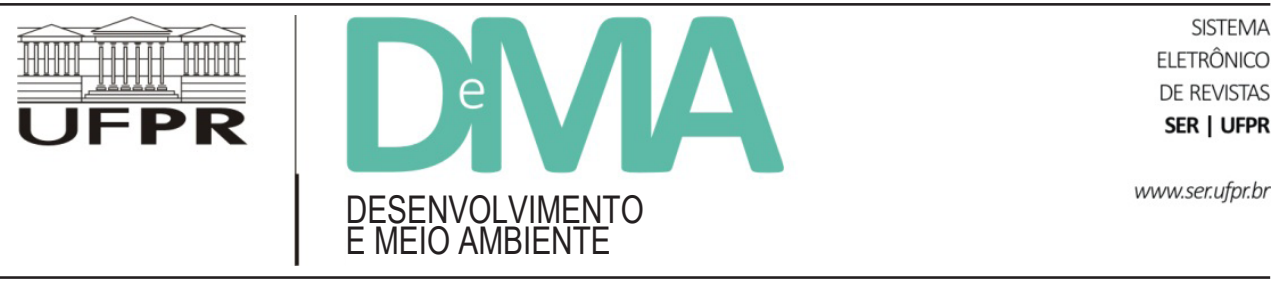

\title{
Caracterización de los Sistemas de Áreas Naturales Protegidas en dos metrópolis Mexicanas
}

\author{
Caracterização de Sistemas de Áreas Naturais Protegidas \\ em duas metrópoles mexicanas
}

\section{Characterization of Protected Natural Areas Systems in Two Mexican Metropolis}

\author{
Gabriela DE LA MORA-DE LA MORA ${ }^{1 *}$ \\ ${ }^{1}$ Centro Regional de Investigaciones Multidisciplinarias (CRIM), Universidad Nacional Autónoma de México (UNAM), Cuernavaca, Morelos, \\ México. \\ *E-mail de contacto: gdlm@correo.crim.unam.mx
}

Artículo recibido el 17 de enero, 2016, versión final aceptada el 7 de julio, 2016.

RESUMEN: A partir de un análisis policéntrico aplicado a nivel mezzo regional se caracterizan los sistemas de áreas naturales protegidas (SANP's) urbanas y periurbanas en las metrópolis mexicanas de Guadalajara y Monterrey. Estos sistemas han sido definidos por la cercanía de las ANP's a las zonas urbanas, más no por una perspectiva normativa o de política pública existente. Las ANP's que conforman ambos sistemas, son administradas y gestionadas por distintos niveles de gobierno (federal, estatal y municipal), los que enfrentan retos importantes en su gestión, ante los procesos de urbanización locales. Siendo uno de los temas más importantes, la falta de coordinación y comunicación entre las autoridades de los distintos niveles de gobierno. El estudio se realiza a partir del análisis de información documental especializada en el tema y datos empíricos recabados por medio de entrevistas a actores sociales clave en cada una de las metrópolis.

Palabras clave: sistemas de áreas naturales protegidas urbanas y periurbanas; sistemas policéntricos; conservación de ANP; servicios ambientales; Guadalajara y Monterrey.

RESUMO: $\quad$ A partir de uma análise policêntrica aplicada a nível mezzo regional, são caracterizados os sistemas de áreas naturais protegidas (SANP) urbanas e periurbanas nas cidades mexicanas de Guadalajara e Monterrey. Estes sistemas são definidos pela proximidade das áreas urbanas e as áreas naturais protegidas, mas não por uma perspectiva normativa ou de política pública existente. As áreas naturais protegidas que fazem parte de ambos os sistemas são administradas e geridas por diferentes níveis de governo (federal, estadual e municipal), que enfrentam grandes desafios na sua gestão, causados por processos de urbanização locais, sendo uma das questões mais importantes a falta de coordenação e comunicação entre as autoridades dos diferentes níveis de 
governo. O estudo é realizado com base na análise de informação documental especializada no assunto e em dados empíricos recolhidos por meio de entrevistas com as principais partes interessadas em cada uma das metrópoles.

Palavras-chave: sistemas de áreas naturais protegidas urbanas e periurbanas; sistemas policêntricos; conservação; serviços ambientais; Guadalajara e Monterrey.

ABSTRACT: From a polycentric analysis applied to mezzo regional level are characterized the urban and peri-urban's protected natural areas systems (PNAS) in the Mexican cities of Guadalajara and Monterrey. These systems have been defined by the proximity of the PNA's to the urban areas, but not by a policy perspective or an existing public policy. The protected natural areas comprised in both systems are administered and managed by different levels of government (federal, state and municipal), which are facing major challenges in their management because of the local urbanization processes. Being one of the most important issues, the lack of coordination and communication between government authorities at different levels. The research is conducted based on the analysis of documentary information specialized in these subjects and empirical data gathered through interviews with key stakeholders in each metropolis.

Keywords: urban and peri-urban Protected Natural Areas Systems; polycentric systems; Protected Natural Areas conservation; environmental services; Guadalajara and Monterrey.

\section{Introducción}

El análisis de las interacciones socio-ecológicas evidencia las distintas concepciones de la relación sociedad-naturaleza que están en acción en un espacio y tiempo específicos. Estas concepciones están en constante transformación recíproca y se manifiestan a través de relaciones interdependientes entre los sistemas naturales y sociales, materializadas en procesos de toma de decisiones vinculados con la gestión del territorio.

A partir del enfoque de gobernanza ambiental multinivel y multi escalar, caracterizamos los Sistemas de Áreas Naturales Protegidas (SANP's) en las zonas urbanas y peri-urbanas de las ciudades mexicanas de Guadalajara y Monterrey. Estos sistemas fueron definidos a partir del planteamiento teórico-metodológico de esta investigación, por lo tanto no existe un mandato normativo o de política pública para ser considerados como sistemas en los procesos de gestión y de toma de decisiones. Sin embargo, se considera la pertinencia de concebir estos sistemas de manera integrada.
Utilizamos el enfoque de sistemas policéntricos para analizar las características generales de ambos SANP's. En los casos de estudio, el crecimiento urbano sin planeación, pone en riesgo la integridad y conservación de ANP's decretadas y administradas por distintos niveles de gobierno (federal, estatal y municipal), lo que tiene repercusiones importantes en la provisión de múltiples servicios ambientales, relevantes para el bienestar de los ciudadanos en las zonas urbanas. Por lo anterior, es necesario que los tomadores de decisiones de los distintos niveles de gobierno y las respectivas sociedades, presten atención de manera conjunta y coordinada, a estos problemas a fin de contribuir al manejo sustentable de los recursos naturales desde una perspectiva de paisaje.

Nuestra hipótesis de trabajo es que la gobernanza vinculada a la conservación de las áreas naturales protegidas ubicadas en las zonas urbanas y la periferia de las ciudades de Guadalajara y Monterrey está fragmentada, pues falta comunicación y coordinación entre los distintos órdenes de gobierno encargados de su gestión; ya que, las autoridades 
locales o estatales no tienen una visión sistémica o regional que permita establecer esquemas de gobernanza integrados.

Es pertinente analizar este fenómeno en un contexto político en el que la toma de decisiones relacionada con la conservación, fomenta la implementación de estrategias de mercado. Este paradigma aplicado a las políticas públicas ambientales en países en desarrollo puede generar mayores inequidades, ya que la creación de nuevos mercados no es trivial ni posible sin la acción de un Estado fuerte y eficiente (Agrawal \& Lemos, 2007; Fletcher, 2010; Norgaard, 2010).

Se trata de una investigación cualitativa, en la que se utilizó el método inductivo-deductivo. Se emplearon diversas técnicas de recolección de datos para responder la hipótesis de investigación tales como: la consulta de fuentes documentales (periódicos y materiales especializados sobre los temas analizados); y entrevistas semi-estructuradas a actores clave vinculados a la gestión y conservación de ANP's en las metrópolis de Guadalajara y Monterrey.

Las categorías de actores clave entrevistados fueron: funcionarios de gobierno encargados directa o indirectamente de la gestión de ANP's federales, estatales y municipales (p.e. directores de áreas naturales protegidas); organizaciones de la sociedad civil organizada formal e informalmente (p.e. organizaciones no gubernamentales formalmente constituidas y asociaciones de personas especializadas en el tema de la conservación o vinculadas con una o más áreas naturales protegidas en las metrópolis analizadas); sector académico y de investigación (investigadores y consultores, que participan en asociaciones civiles y que influyen en la toma de decisiones); otros actores (como la iniciativa privada).
En total, se realizaron cincuenta y ocho entrevistas. Veintiocho en la metrópoli de Guadalajara durante noviembre de 2013, enero de 2014 y enero y febrero de 2015. Mientras que en Monterrey se realizaron treinta entrevistas a lo largo de 2012 y en febrero de 2015. La información de las entrevistas se sistematizó empleando el software de análisis cualitativo Atlas.Ti (versión 1.0.24).

El presente trabajo se divide en tres partes. En la primera se discuten y definen los conceptos utilizados en el análisis de la gobernanza policéntrica. En la segunda parte, se caracteriza brevemente el surgimiento de la política de conservación de áreas naturales protegidas en México y a nivel internacional. Y en la tercera parte se estudian los rasgos generales de los sistemas de ANP's en las metrópolis de Guadalajara y Monterrey.

\section{Sistemas policéntricos y SANP'S}

El término policentrismo es un concepto difuso y polivalente (Burger \& Meijers, 2012; Vasanen, 2012). Ha sido utilizado por diversas disciplinas de las ciencias sociales para referirse a la morfología y funcionalidad de los sistemas urbanos (van Meeteren et al., 2015, p. 4). El uso del concepto es problemático por el amplio número de propiedades (intenciones) que se le adscriben. Además, existe una creciente cantidad de situaciones en las que se aplica (extensión) y es replicado por diferentes autores en distintos contextos (Sartori, 1970, citado en Van Meeteren et al., 2015).

Ostrom et al. (1961) utilizaron el concepto de policentrismo para analizar la interacción de una amplia gama de organismos públicos y privados dedicados al suministro y producción de servicios públicos en las áreas metropolitanas en Estados Unidos (Ostrom, 2009, p. 3). Entre otros hallazgos, 
señalaron que en la medida en que todas estas unidades se tomen en cuenta entre sí en un marco de relaciones competitivas, se pueden establecer compromisos contractuales, esquemas de cooperación o mecanismos de resolución de conflictos. De esta forma, las diversas jurisdicciones políticas en un área metropolitana pueden funcionar de manera coherente y con ciertos patrones de comportamiento (Ostrom, 1961, p. 831-32, citado en Ostrom, 2009, p. 3), lo que implicaría la existencia de un sistema policéntrico funcionalmente hablando, en el que se toman decisiones que conciernen a un territorio independientemente de las jurisdicciones político-administrativas.

Entonces, la policentricidad supone la existencia de múltiples espacios de toma de decisiones formalmente independientes entre sí, en los que están presentes múltiples actores, quienes de manera autónoma toman decisiones y al mismo tiempo, generan las condiciones necesarias para colaborar entre sí (Ostrom et al., 1961, p. 831 citado en Douglas \& Dietz, 2011, p. 190). La gobernanza ambiental idealmente debería construirse y mantenerse a partir de un esquema policéntrico en la toma de decisiones tomando en cuenta dos componentes:

- La gobernanza multinivel, sustentada en las relaciones [institucionalizadas o no] que vinculan y coordinan acciones de diversos agentes públicos y privados, fomentando inclusivamente intercambios y colaboración entre ellos (Peters \& Pierre, 2004, p. 77-79, 81).

- La gobernanza multiescala, sustentada en unidades políticas delimitadas y organiza- das (que pueden referir a provincias, naciones, entidades regionales y supranacionales, etc.) que mantienen vínculos institucionales (constitucional y estatuariamente). Se trata de procesos sociopolíticos que operan en diversas jurisdicciones (Buizer et al., 2011).

En esta investigación entendemos a la gobernanza ambiental como el "proceso de formulación y refutación de imágenes, diseños y ejecución de los procedimientos y prácticas que configuran el acceso, control y uso de los recursos naturales entre actores diferentes" (De Castro et al., 2015, p. 18). Implica la adaptación o la generación de nuevas estructuras de gobernanza sustentadas en coaliciones y luchas sociales locales (Soja, 2004; Konijnendijk, 2015).

El planteamiento de los sistemas policéntricos ha sido utilizado para comprender el manejo de recursos de uso común en pequeña escala; sin embargo, se reconoce la necesidad de utilizar este análisis a escalas más amplias (Gruby \& Basurto, 2013, p. 260; Heikkila et al., 2011; Ostrom, 2009). En ese sentido, este estudio adopta un enfoque regional mezzo. Según Soja (2004) el enfoque sintetiza creativamente espacios macro y micro, que permiten observar al territorio como un elemento dinámico, contraponiéndose a las antiguas definiciones y formas tradicionales de aplicar las políticas en que se concebía al territorio como un elemento fijo y estático. El uso de este enfoque permitirá determinar si existen morfológicamente (o en términos de contigüidad) y funcionalmente (en términos político-administrativos) un SANP's en dos de las zonas metropolitanas ${ }^{1}$ más importantes en México.

\footnotetext{
Se entiende por zona metropolitana a aquellas ciudades que, independientemente de su tamaño, rebasan sus límites territoriales político-administrativos para conformar un área urbana ubicada en dos o más municipios. La metropolización de una ciudad tiene lugar cuando, en su proceso de expansión utiliza para el desarrollo urbano suelo que pertenece a uno o más municipios en los cuales se ubica la ciudad central (Sobrino, 2003. p. 461).
} 
El análisis sobre la existencia o no de un sistema de áreas naturales protegidas en las metrópolis de estudio, busca indagar sobre la existencia de vínculos entre tomadores de decisiones de distintas escalas y niveles, quienes pueden articular y estructurar morfológica y funcionalmente diversas unidades socio-territoriales para lograr un funcionamiento más eficiente e integrado del sistema, es decir, entre los sistemas urbanos y naturales en interacción.

La consolidación de estos sistemas de SANP's en las metrópolis de Guadalajara y Monterrey es relevante para la provisión y mantenimiento de múltiples servicios ambientales, especialmente los hidrológicos, en esas zonas urbanas. Existen evidencias científicas que demuestran que áreas forestales en interacción con zonas urbanas permiten mejorar la calidad y cantidad de agua, entre otros servicios ambientales, siendo un aspecto clave para fomentar su declaratoria (Dudley \& Stolton, 2003; Stolton \& Dudley 2007; Comberti et al., 2015).

A continuación, se analizan las características generales de la política de áreas naturales protegidas desde su origen y su vínculo con el desarrollo de las metrópolis, lo que servirá de base para proponer la conformación de los SANP's en las metrópolis de Guadalajara y Monterrey, tema que se abordará en el último apartado.

\section{Conservación de ANP's y su importancia para las metrópolis}

La conformación de jure o de facto de los SANP's en México en sus distintas regiones, es resultado de un esfuerzo sostenido por parte de autoridades gubernamentales de los distintos niveles de gobierno (nacional, estatal y municipal) propietarios y sociedades locales. Estos esfuerzos no han sido ajenos a los conflictos sociales, ya que las declaratorias implican una transformación en los derechos de acceso a los recursos naturales, afectando intereses de actores locales.

El gobierno federal inició oficialmente la política de conservación de áreas naturales protegidas en 1898 con el decreto del Bosque Nacional el Monte Vedado del Mineral El Chico, en el estado de Hidalgo, localizado en el centro del país (Simonian, 1999, p. 85-86; De la Maza et al., 2003, p. 18).

En un inicio, los criterios para decretar las distintas categorías de protección no tenían necesariamente un sustento técnico científico, sino que estaban relacionados con la belleza escénica, el potencial recreativo y el valor ecológico de los ecosistemas (Simonian, 1999, p. 124). Los límites de los terrenos y las áreas, se definían por medio de rasgos fisiográficos y naturales notables, y no a través de coordenadas geográficas claramente definidas, como ocurre actualmente. Estos rasgos se evidenciaron en las primeras declaratorias de Parques Nacionales a nivel internacional, siendo el primer caso el Parque Nacional de Yellowstone en Estados Unidos creado en 1887; pero también este fenómeno se presentó en la Reserva Perito Moreno en Argentina en 1903; la Reserva Forestal Malleco en Chile en 1907 (Primack et al., 2001); el Parque Imperial Nikko en Japón en 1911; La Bralowieza en Suiza en 1914, entre otros (Hesselbach \& Pérez, 1996; De la Mora-De la Mora, 2004).

El establecimiento de parques nacionales y reservas forestales permeó diferenciadamente en México, predominando declaratorias promovidas por el gobierno federal a lo largo del siglo XX. Éstas generalmente fueron percibidas por los habitantes locales como una imposición, ya que muchos decretos se hicieron sin tomar en cuenta sus necesidades, lo que provocó que no se respetaran las nuevas formas de uso de los recursos impuestas. 
Paralelamente, las políticas de desarrollo rural pasaron por alto la conservación de los recursos forestales, ya que en muchos casos se llegaron a considerar tierras ociosas o baldias por no tener un propósito productivo. Otro fenómeno que tuvo lugar, fue la concesión de terrenos forestales a empresas madereras que realizaban intensas extracciones comerciales. En teoría el propósito de las concesiones era incorporar áreas marginales campesinas e indígenas al desarrollo industrial del país. Muchas de esas concesiones duraron en promedio 25 años, pero hubo casos que alcanzaron hasta 60 (Gerez, 2001; Merino, 2004). Asimismo, se declararon vedas forestales, lo que en algunos casos evitó la desaparición de importantes zonas forestales, pero en otros fomentó el saqueo y el uso ilegal de los recursos naturales.

El resultado fue que en los años 1960 se aceleró la pérdida del patrimonio natural de México por múltiples factores, entre ellos los conflictos agrarios, los programas de colonización, la creciente presión demográfica y la puesta en marcha de proyectos agropecuarios poco sostenibles (De la Mora-De la Mora, 2004).

En la primera mitad del siglo XX, el gobierno central impulsó decretos de ANP's en zonas forestales relevantes para centros urbanos. La intención era proteger cuencas claves para preservar los servicios ambientales hidrológicos para usos urbanos y agrícolas. Muchos de esos decretos no operaron ni se cumplieron, ya que como se mencionó antes, generalmente los propietarios de estas tierras no participaron ni en la conceptualización ni en la operación de las áreas y en los casos en que se expropiaron las tierras, no siempre fueron indemnizados. Además, durante décadas no se asignaron recursos financieros o éstos fueron insuficientes para operar y gestionar las ANP's; asimismo, no se contaba con recursos humanos, ni capacidades técnicas para hacer efectiva su operación (Bello, 2013, p. 358).

Pese a las limitaciones mencionadas, tanto el gobierno federal, como los estatales y en menor medida los municipales continuaron estableciendo ANP's en distintas regiones del país. En México, actualmente los decretos de protección generados por los distintos niveles de gobierno no son la única modalidad de conservación reconocida. Existen predios voluntariamente protegidos por propietarios privados (personas físicas y morales, comunidades y ejidos) que son certificados por el gobierno federal. De las poco más de 30 millones de hectáreas que están bajo alguna categoría de protección a nivel nacional $85 \%$ es administrada por el gobierno federal, $13 \%$ por gobiernos de los estados, $0.5 \%$ por gobiernos municipales o locales y $1.3 \%$ son áreas de protección voluntaria. En suma, aproximadamente $15 \%$ de la superficie terrestre del país se encuentra protegida por alguna de esas modalidades ${ }^{2}$.

Bezaury-Creel \& Gutiérrez (2009, p. 398) mencionan que el establecimiento de muchas de esas áreas es resultado de esfuerzos casuísticos

\footnotetext{
2 Para hacer el cálculo se utilizaron distintas fuentes en el caso de las ANP federales, la fuente es la Comisión Nacional de Áreas Naturales Protegidas, consulta 7 de octubre de 2015, www.conanp.gob.mx. En el caso de las ANP's estatales, la fuente es la Red Nacional de Sistemas de Áreas Naturales Protegidas. http:/www.anpsestatales.mx/anps.php consulta 7 de octubre de 2015. En el caso de las ANP's municipales la fuente es Bezaury-Creel J.E., J. Fco. Torres, L. M. Ochoa-Ochoa, Marco Castro-Campos, N. Moreno. (2009). Base de Datos Geográfica de Áreas Naturales Protegidas Municipales de México - Versión 2.0, Julio 31, 2009. The Nature Conservancy / Comisión Nacional para el Conocimiento y Uso de la Biodiversidad / Comisión Nacional de Áreas Naturales Protegidas. 2 Capas ArcGIS 9.2 + 2 Capas Google Earth KMZ + 1 Archivo de Metadatos Word. En el caso de las ANP's voluntarias la fuente es la Base de datos en formato excel "Listado de Áreas Destinadas Voluntariamente a la Conservación”. Dirección General de Conservación para el Desarrollo. Comisión Nacional de Áreas Naturales Protegidas. Última modificación 14 de diciembre de 2015 www.conanp.gob.mx Consultado el 22 de diciembre de 2015.
} 
efectuados por diversas administraciones gubernamentales, con diferentes mandatos, orientados a proteger una gran diversidad de atributos del medio natural. Los autores precisan que en general no existía una estrategia clara de conservación.

Actualmente el objetivo estratégico de la conservación de ANP's es mantener la representatividad de los ecosistemas y su biodiversidad, con el fin de asegurar la provisión de los servicios ambientales, mediante la conservación y el manejo sustentable; además de fomentar el desarrollo de actividades productivas en las comunidades que viven en dichas áreas. Por mandato legal, el objetivo del SANP's es fortalecer los vínculos entre las ANP's existentes para lograr su adecuado manejo. La creación y consolidación de este sistema sobre todo ha sido promovida para las áreas administradas por el gobierno federal en los distintos estados de la República Mexicana, pero también se busca fomentar la participación de otras ANP's administradas por los gobiernos de los estados y municipios.

Institucionalmente, la formación de un SANP's a nivel nacional es parte de un complejo e inacabado proceso que inició formalmente desde la promulgación de la Ley General del Equilibrio Ecológico y la Protección al Ambiente (LGEEPA) en 1988. En esta ley se define la conformación del Sistema Nacional de Áreas Naturales Protegidas como la "integración" de las ANP's de competencia federal a partir de ciertos criterios (LGEEPA, Sección IV) y señala la necesidad de lograr la convergencia en las acciones y formas de intervención de distintas entidades de la administración pública federal y autoridades estatales, con la finalidad de que se acaten los programas y acciones que afectan esos territorios ${ }^{3}$. Asimismo, la LGEEPA establece el marco general para que los estados y municipios elaboren sus propias regulaciones en la materia, las que tienden a ser un "espejo de la legislación federal" (Bezaury-Creel \& Gutiérrez, 2009 , p. 415). En la mayoría de los estados aún no existe una política manifiesta con respecto a la conservación de las ANP's, más bien prevalece el sello personal que imprimen las autoridades locales en función de sus intereses y sensibilidad sobre el tema (Bezaury-Creel \& Gutiérrez, 2009, p. 415). Los SANP's analizados en este estudio se inscriben en esa dinámica fragmentada, en la que además los tomadores de decisiones locales no han incorporado en su agenda política de conservación la gestión de las distintas áreas naturales protegidas como parte de un sistema.

Por otro lado, los gobiernos municipales tienen la facultad de "autorizar, controlar y vigilar la utilización del suelo en el ámbito de su competencia, en sus jurisdicciones territoriales" y "otorgar licencias y permisos para construcciones". Al mismo tiempo en estas áreas el gobierno federal se encarga de calificar el impacto ambiental que los cambios de uso de suelo pueden causar en el ANP (Bezaury-Creel \& Gutiérrez, 2009, p. 406). Lo anterior implica confrontaciones entre los distintos órdenes de gobierno en los procesos de toma de decisiones, así como entre el interés público y

\footnotetext{
Esta ley define las categorías de manejo y formas de delimitación territorial de las ANP. Asimismo, se reconoce que los gobiernos estatales y municipales pueden establecer sus propias categorías de protección que estarán bajo su adscripción, tales como parques y reservas estatales, entre otras que establezcan las legislaciones estatales. En tanto que los gobiernos municipales pueden establecer zonas de conservación ecológica municipal y otras modalidades de conservación y manejo que ellos establezcan en sus respectivas legislaciones. En particular, las categorías comprendidas en la LGEEPA (Artículo 46) bajo competencia de la federación son: reservas de la biósfera, parques nacionales, monumentos naturales, áreas de protección de recursos naturales, áreas de protección de flora y fauna, santuarios y áreas destinadas voluntariamente a la conservación.
} 
el privado (ver Bezaury-Creel \& Gutiérrez, 2009, p. 418$)^{4}$.

La red actual de ANP's es insuficiente para conservar adecuadamente una porción representativa de la biodiversidad de México (Bezaury-Creel \& Gutiérrez, 2009, p. 426; Conanp, 2014, p. 13). Sin embargo, al menos en la agenda política federal existe mayor claridad para adoptar una perspectiva de manejo integrado del paisaje a fin de reducir la fragmentación y el aislamiento de los ecosistemas en el largo plazo, la que ha sido integrada en la Estrategia de la Comisión Nacional de Áreas Naturales Protegidas 2040. De esta forma, la conservación se integra a los usos del suelo, dejando de manifiesto la interdependencia de las unidades del paisaje (Conanp, 2014, p. 18).

En el siguiente apartado se caracterizan los SANP's de las metrópolis de Guadalajara y Monterrey y algunas generalidades sobre los mismos.

\section{Caracterización de los SANP's de Guadalajara y Monterrey}

México es un país eminentemente urbano, $78 \%$ de la población vive en ciudades. En 2010, en el país se contabilizaron 59 zonas metropolitanas donde reside el $57 \%$ de la población. Las ciudades de Guadalajara y Monterrey son las capitales de los estados de Jalisco y Nuevo León respectivamente. Por su relevancia demográfica y económica, tradicionalmente han ocupado los primeros lugares en la lista de metrópolis mexicanas, siendo antecedidas sólo por la Ciudad de México, que es la capital de la República Mexicana. El desarrollo de ambas metrópolis se empezó a consolidar durante el periodo de industrialización sustitutiva de importaciones (Negrete, 2010, p. 188). Actualmente ocupan el segundo y tercer lugar en importancia por el número de habitantes a nivel nacional ${ }^{5}$; concentran $61 \%$ y $89 \%$ de la población de la entidad en sus ciudades capitales, respectivamente. Asimismo, ocupan el cuarto y tercer lugar por su participación en la generación de riqueza económica a nivel nacional aportando 6 y 7\% del Producto Interno Bruto (PIB), respectivamente.

En ambas metrópolis el número de declaratorias de ANP's registra un mayor desarrollo a partir de los años 2000, ya que el $90 \%$ de las áreas fueron decretadas entre 2000 y 2014. Muchas de estas ANP's al estar ubicadas cerca o dentro de ciudades, están en riesgo de que su superficie se degrade o disminuya como a consecuencia del crecimiento de la mancha urbana.

Los sistemas de ANP's de Guadalajara y Monterrey analizados, fueron definidos a partir de la cercanía que las áreas registran a las zonas urbanas, más no porque formen parte de una política estratégica de conservación, que incluya las áreas decretadas a nivel municipal, estatal y federal. La gestión de las áreas que forman parte de estos sistemas responde a una lógica de fragmentación, debido a que las autoridades encargadas de su administración, desarrolla acciones aisladamente respecto a sus homólogos de los otros niveles de gobierno.

\footnotetext{
4 Bezaury-Creel \& Gutiérrez (2009, p. 418) citan diversos casos sobre resoluciones judiciales que revocan decretos de ANP y controversias constitucionales sobre autorizaciones de desarrollos urbanos en ANP's federales, contraponiendo las facultades federales versus las municipales en las ANP's.

5 El Censo General de Población de 2010 contabilizó 4.5 millones de habitantes para Guadalajara y su áreas metropolitana y Monterrey 4.1 millones de habitantes.
} 
En el caso de Guadalajara el sistema se compone de siete áreas naturales protegidas que abarcan poco más de 84 mil hectáreas. Se trata de un Área de Protección de Flora y Fauna conocida como Bosque La Primavera, que es co-administrada por el gobierno federal y el gobierno estatal; cinco ANP's administradas por los municipios, entre ellas, la Zona de Conservación Ecológica Barranca de Oblatos-Huentitán; y las Áreas Municipales de Protección Hidrológica Barranca del Río Santiago, Bosque Los Colomos, Bosque El Nixticuil-San Esteban-El Diente y Los Colomos III; y un área estatal de Protección Hidrológica denominada Cerro Viejo-Chupinaya-Los Sabino (Ver Figura 1). En el caso específico de la ciudad de Guadalajara, los tres niveles de gobierno están presentes en

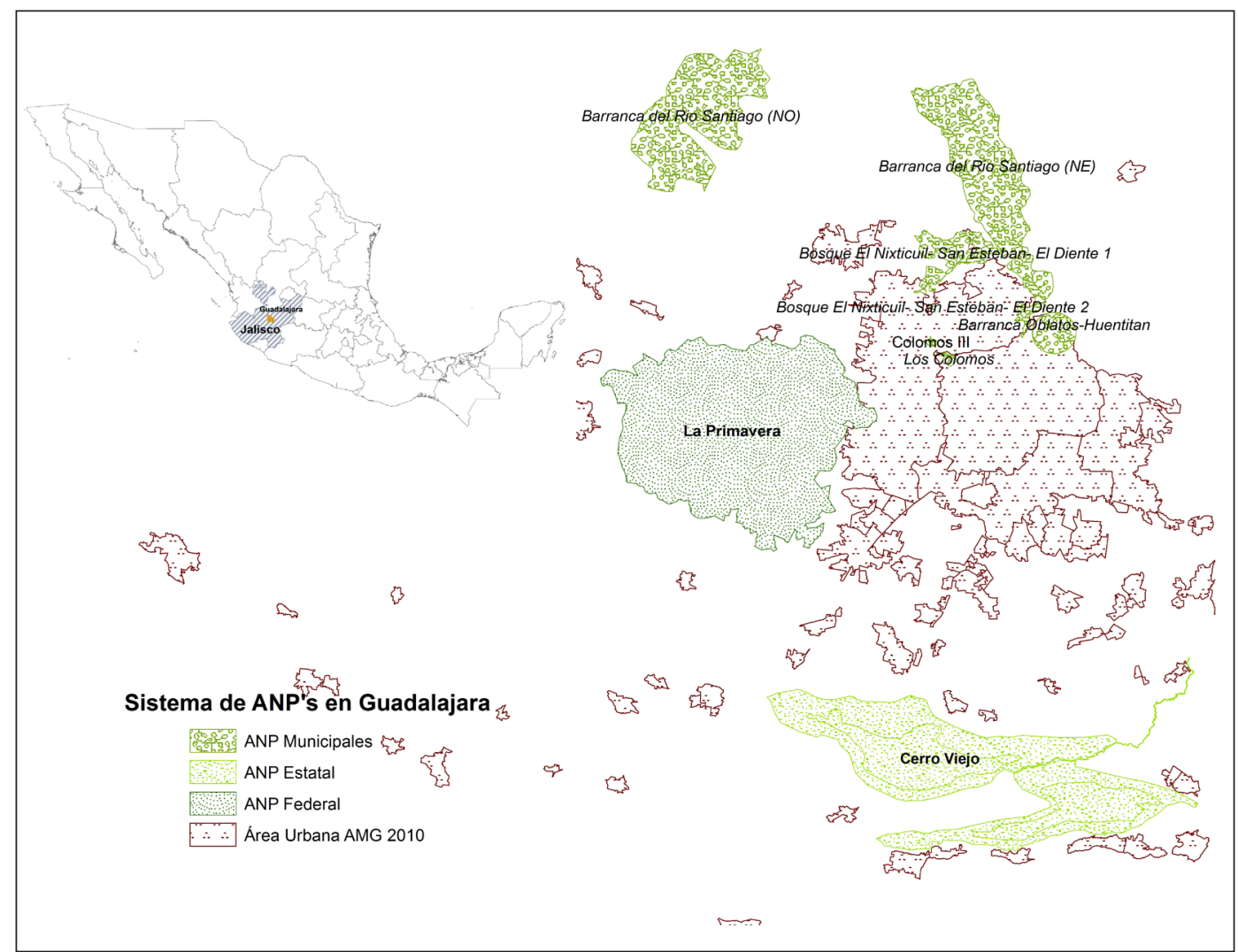

FIGURA 1 - Sistema de ANP's en Guadalajara.

FUENTE: Elaboración propia ${ }^{6}$.

\begin{abstract}
6 Con información de la Secretaría de Medio Ambiente y Desarrollo Territorial del Estado de Jalisco (2015) correspondiente a las áreas naturales protegidas municipales; Bezaury-Creel J.E., J. Fco. Torres, L. M. Ochoa-Ochoa, Marco Castro-Campos, N. Moreno. (2009). Base de Datos Geográfica de Áreas Naturales Protegidas Estatales, del Distrito Federal y Municipales de México - Versión 2.0, Julio 31, 2009. The Nature Conservancy / Comisión Nacional para el Conocimiento y Uso de la Biodiversidad / Comisión Nacional de Áreas Naturales Protegidas. 2 Capas ArcGIS 9.2 + 2 Capas Goggle Earth KMZ + 1 Archivo de Metadatos Word; Información de Áreas Naturales Protegidas Federales, Comisión Nacional de Áreas Naturales Protegidas 2014. 09/01/2014; los polígonos del Área Urbana corresponde a información del INEGI para los respectivos años.
\end{abstract}


los procesos de gestión de las áreas de conservación. En particular, $25 \%$ de la superficie protegida está bajo resguardo de gobiernos municipales, $37 \%$ es co-administrado por la federación y el gobierno del estado, y 38\% por el gobierno del Estado de Jalisco (Ver Figura 2).

\section{Administración de la Superficie de ANP en Guadalajara}

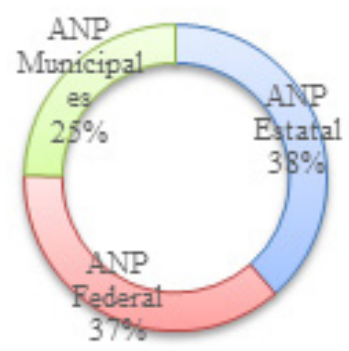

FIGURA 2 - Administración de la Superficie de ANP's en Guadalajara.

FUENTE: Elaboración propia.

En conjunto forman parte del SANP's, que aunque no funciona política y administrativamente como tal, actores de la sociedad civil reconocen su valor ecológico y social por los beneficios que aportan a la sociedad en su conjunto.

Varias de las ANP's de este sistema, han sido promovidas por la sociedad civil a través de un proceso de lucha que ha implicado la movilización de personas y organizaciones para defender espacios públicos (decretados o no), que son vitales para la producción y mantenimiento de servicios ambientales para la ciudad. Especialmente nos referimos a las declaratorias del Bensedi ${ }^{7}$, Colomos III y Barranca de Huentitán, en los que la sociedad, es decir, vecinos y organizaciones de la sociedad civil, solicitaron a sus autoridades municipales (Zapopan para el caso de las dos primeras y la última de Guadalajara) que se declaren esos espacios como ANP's para evitar que la voracidad inmobiliaria los desaparezca. En todos los casos las movilizaciones sociales lograron consolidar las declaratorias, y su lucha aún continúa.

En Guadalajara no existe un organismo especializado que posibilite procesos de toma de decisiones policéntricos o sistémicos en materia de conservación. Quizá uno de los esfuerzos más importantes en este sentido es el caso del Organismo Público Descentralizado (OPD) creado específicamente para el Bosque La Primavera en 2015. El propósito de esta entidad es fortalecer al organismo administrador del área a fin de evitar que los procesos de toma de decisiones del ANP favorecieran a actores específicos (p.e. propietarios o director del área) (Entrevista Personal, JDV, 2015). Para ello, se formó una Junta de Gobierno en la que participan veintiún actores del sector público y civil, así como representantes de los tres niveles de gobierno. Este esfuerzo se vio limitado inicialmente porque los propietarios de los terrenos de esa ANP manifestaron su disgusto ante la baja representación que tenían en dicho órgano, argumentando que ellos eran mayoría. En contraposición, constituyeron un Consejo Regulador, siendo una medida de rechazo al modelo de gobernanza planteado por el OPD (Cárdenas, 2014).

Paralelamente, organizaciones de la sociedad civil han propuesto mantener y generar corredores biológicos, así como generar un cinturón verde alrededor de la ciudad, que permita asegurar la provisión de servicios ambientales (Entrevistas Personales, ACJ, 2015; MS, 2015 y SG, 2015). Quizá este interés podría derivar en un futuro en la consolidación de

\footnotetext{
Para el caso del Bensedi, se puede consultar el artículo De la Mora y Montaño (2016).
} 
espacios de toma de decisiones de carácter policéntrico para el área metropolitana de Guadalajara, ya que crecientemente se reconoce la importancia de estas zonas para generar servicios ambientales.

En el caso de Monterrey, el sistema está compuesto por doce áreas que comprenden una superficie de casi 310 mil hectáreas. Se trata de dos áreas bajo administración de la federación: el Parque Nacional Cumbres de Monterrey y el Monumento
Natural Cerro de la Silla; y diez ANP's protegidas por el gobierno estatal, es decir, las Zonas Sujetas a Conservación Ecológica Sierra Las Mitras, Sierra El Fraile y San Miguel, Sierra Corral de los Bandidos, Sierra Cerro de la Silla, Cerro La Mota, Cerro El Topo y Sierra Picachos; el Parque Urbano Cerro del Obispado, el Parque Urbano Lineal Río Santa Catarina y el Parque Ecológico La Pastora (Ver Figura 3).

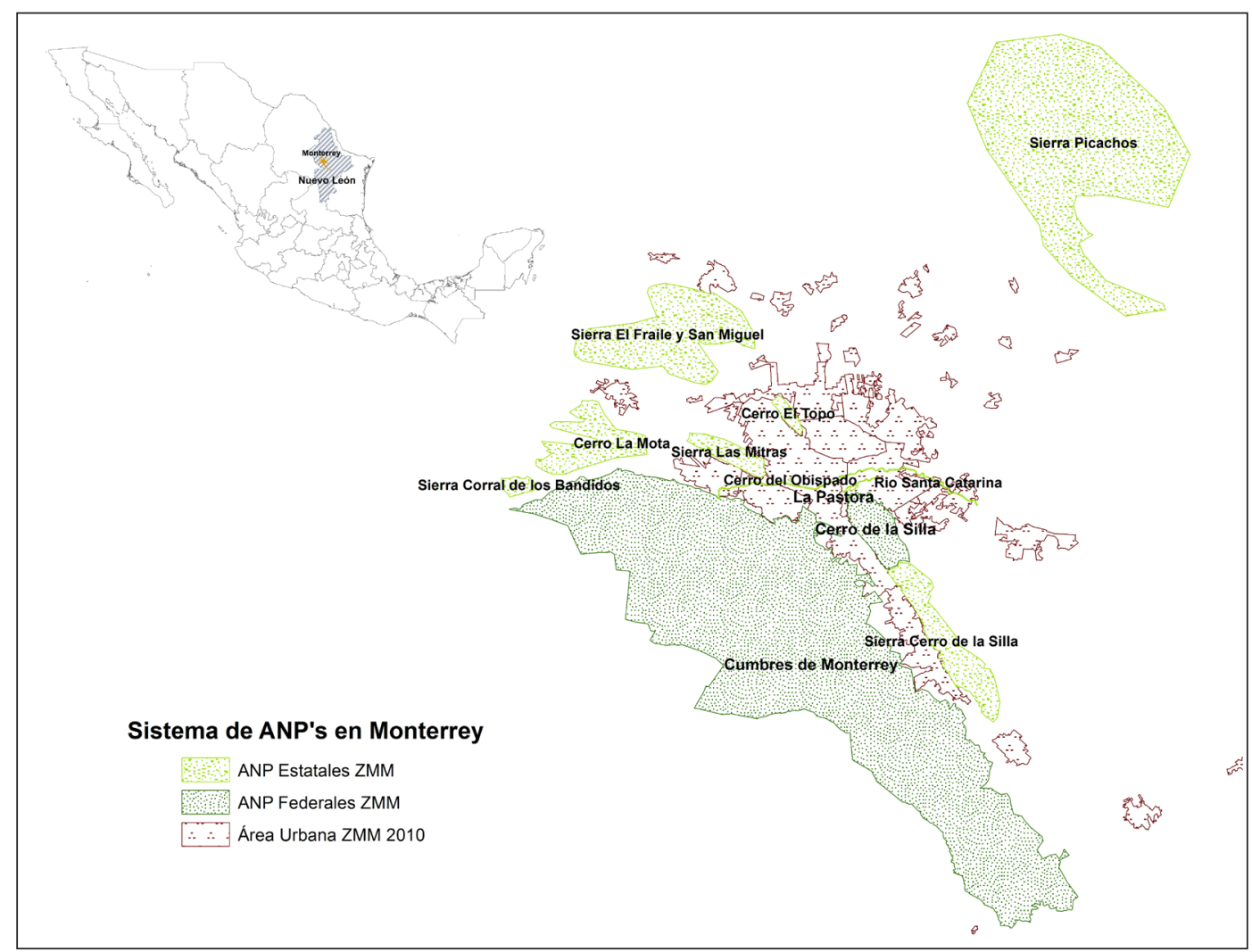

FIGURA 3 - Sistema de ANP en Monterrey.

FUENTE: Elaboración propia ${ }^{8}$.

\footnotetext{
8 Con información de Parques y Vida Silvestre del Gobierno del Estado de Nuevo León, polígono del PNCM 1939; Bezaury-Creel J.E., J. Fco. Torres, L. M. Ochoa-Ochoa, Marco Castro-Campos, N. Moreno. (2009). Base de Datos Geográfica de Áreas Naturales Protegidas Estatales, del Distrito Federal y Municipales de México - Versión 2.0, Julio 31, 2009. The Nature Conservancy / Comisión Nacional para el Conocimiento y Uso de la Biodiversidad / Comisión Nacional de Áreas Naturales Protegidas. 2 Capas ArcGIS 9.2 + 2 Capas Goggle Earth KMZ + 1 Archivo de Metadatos Word; Información de Áreas Naturales Protegidas Federales, Comisión Nacional de Áreas Naturales Protegidas 2014. 09/01/2014; los polígonos del Área Urbana corresponde a información del INEGI para los respectivos años.
} 
El cuarenta y uno por ciento de la superficie de ANP's es administrada por el gobierno del estado de Nuevo León y 59\% por el gobierno federal (Figura 4).

\section{Administración de la Superficie de ANP en Monterrey}

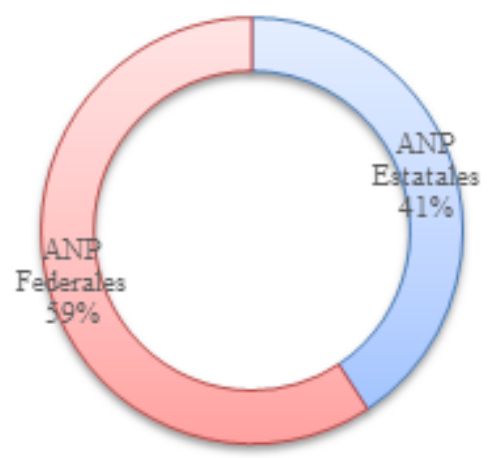

FIGURA 4 - Administración de la Superficie de ANP's en Monterrey.

FUENTE: Elaboración propia.

Siete las ANP's localizadas cerca de la metrópoli y que se encuentran bajo resguardo del gobierno del estado de Nuevo León, fueron decretadas junto con otras dieciséis áreas ubicadas en otras regiones de la entidad, en un solo acto jurídico en el año 2000. El origen de esta iniciativa está asociada a la participación del sector académico, quienes en alianza con organizaciones de la sociedad civil contribuyeron a la creación de nuevas ANP's (Bello, 2013, p. 359). Un grupo de investigadores de la Universidad Autónoma de Nuevo León y del Instituto de Tecnológico y de Estudios Superiores de Monterrey, entre otras universidades locales, participaron en la elaboración de estudios en diferentes regiones del estado para determinar las áreas susceptibles a ser decretadas por su representatividad, relictualidad e integridad ecológica. Los resultados obtenidos fueron utilizados como justificación técnica y científica para generar veintitrés decretos de áreas naturales protegidas, no sólo en la zona metropolitana, sino en todo el estado.

Lo anterior permitió dar cumplimiento al mandato de la Ley Ambiental de Nuevo León, que estipula que el gobierno del estado se encargaría de establecer y administrar las ANP's del estado además de coordinar el Sistema Estatal (Ley Ambiental del Estado de Nuevo León, Art. 1, Fracc. IV). Este esfuerzo se llevó a cabo durante el periodo de gobierno 1997-2003 durante el cual el gobierno de Nuevo León invirtió recursos, contrató personal, asignó vehículos e hizo crecer el sistema de ANP'S nombrando estratégicamente personal en las distintas áreas (Entrevista Personal, CCA, 2015). Estas acciones le valieron a la entidad para que ocupara un lugar ejemplar a nivel nacional, por el avance decido en la consolidación de su SANP's. Sin embargo, para las subsecuentes administraciones la conservación dejó de ser un tema relevante. Se evidenció el desmantelamiento del sistema y se revirtieron los avances logrados, lo que se tradujo en una reducción presupuestal y en el número de personas dedicadas a trabajar en el sistema. Esta acción evidencia que el desarrollo y cumplimiento de la política de conservación es, entre otras cosas, un acto de voluntad política, más que un mandato jurídico o una necesidad social traducida en una política pública. El SANP's estatal existe, pero en los hechos no funciona como tal, ni tampoco existe un mecanismo institucional que favorezca los procesos de toma de decisiones de manera conjunta.

Al igual que en Guadalajara, la participación de la sociedad civil organizada, ha jugado un papel fundamental en los procesos de toma de decisiones para tratar de mantener la integridad de algunas ANP's del sistema. Entre los casos más emblemáticos registrados están la defensa de la zona conocida 
como Valle de Reyes que forma parte del Parque Nacional Cumbres de Monterrey en donde la empresa inmobiliaria Dos Carlos pretendía iniciar un proyecto de urbanización, lo que generó protestas de organizaciones de la sociedad civil y grupos sociales. Sin embargo, la inmobiliaria promovió un amparo entre 2000 y 2002 que derivó en una resolución judicial en la que se dejó sin efecto el decreto de ANP en esa porción de terreno, quedando éste como una isla dentro del Parque rodeado de un territorio bajo protección (Bello, 2010, p. 365). Otro caso es el Parque Ecológico La Pastora que en septiembre de 2008 la empresa Desarrollo Deportivo y Comercial S.A. de C.V. subsidiaria de Fomento Económico Mexicano (FEMSA) que históricamente ha estado ligada al negocio de bebidas (cerveza, refresco y agua embotellada) anunció la posibilidad de construir un nuevo estadio de fútbol en una porción del parque que primeramente fue desincorporada (Entrevista Personal GMB, 2016; Foforocha, 2010; Campos, 2011). Después de dos años y medio de movilizaciones y protestas sociales de diversos colectivos en contra del proyecto, así como la emisión de opiniones técnicas y científicas en contra de la construcción del estadio, ésta no se logró detener, siendo inaugurado en agosto de 2015.

La experiencia de Monterrey muestra que la gobernanza ambiental vinculada a la conservación de las ANP's depende de la voluntad política en los procesos de toma de decisiones a favor de la conservación. Sin embargo, la construcción de una agenda ambiental y de conservación de ANP's a través de un proceso participativo entre autoridades, sociedad civil, empresarios y científicos ha tenido algunos logros, pero también fracasos y esfuerzos frustrados.

\section{Conclusiones}

Los sistemas de áreas naturales protegidas de Guadalajara y Monterrey que hemos analizado, han sido definidos morfológicamente por la cercanía de estas áreas con los espacios urbanos. Sin embargo, en los procesos de toma de decisiones no existen aún mecanismos que funcionalmente reconozcan a este grupo de áreas como parte de un sistemas ecológico inserto en un contexto urbano. Sin embargo, es importante hacer notar que en el caso de Guadalajara existe un grupo de actores de la sociedad civil que promueve la idea de generar mecanismos metropolitanos para fomentar la conservación de ANP's de manera integrada y con ello favorecer el mantenimiento de diversos servicios ambientales, especialmente los hidrológicos.

Los retos que enfrentan los encargados de gestionar estas áreas son múltiples. La compleja relación entre sociedad y naturaleza en contextos de expansión urbana sin una adecuada planeación territorial, tiene manifestaciones en múltiples niveles y escalas, tanto en lo social, lo político, lo económico, la seguridad, la salud humana y el ambiente. El riesgo de cambio de uso de suelo en esas áreas es uno de los problemas más apremiantes, ya que se les ha considerado como espacios "disponibles" para el crecimiento urbano. Lo que está afectando la integridad y estado de conservación de las ANP's y la provisión de servicios ambientales indispensables para los habitantes de las ciudades. Es importante revertir esta dinámica y sobre todo concebir a estos espacios naturales de mayores dimensiones como parte indisociable de las zonas urbanas.

Los procesos que derivaron en la declaratoria de las áreas naturales protegidas que componen los sistemas de Guadalajara y Monterrey no presentan un patrón homogéneo ni coincidente en términos 
temporales en ambos casos de estudio. Por otro lado, si diferenciamos el nivel de gobierno que interviene en la administración y gestión de cada una de estas áreas, es posible observar que las ANP's administradas por el gobierno federal suelen contar con más recursos financieros, institucionales y humanos para su gestión. Asimismo, son relativamente más estables en términos institucionales a lo largo del tiempo y forman parte de un sistema más amplio en el que participan otras áreas naturales protegidas por la federación en otros estados de la República.

Mientras que en el caso de las ANP's de carácter estatal, éstas presentan una mayor inestabilidad institucional relativa respecto a la federación en términos financieros y humanos a lo largo del tiempo; tal y como lo evidencia el caso de Monterrey.

Mientras que las ANP's gestionadas y administradas por los gobiernos municipales son las que muestran mayor debilidad institucional, ya que los procesos de toma de decisiones están más expuestos a las visiones e intereses políticos de los dirigentes en turno, cuyos periodos de gobierno son más cortos (tres años), y los presupuestos tienden

\section{Referencias}

Agrawal, A.; Lemos, M. C. A greener revolution in the making? Environmental governance in the $21^{\text {st }}$ Century. Environment, 49(5), 36-45, 2007.

Bello, S. M. L. Marco jurídico del Parque Nacional Cumbres de Monterrey. En: Cantú, A. C.; Rovalo M.; Marmolejo J.; Sadot O. H.; Seriñá G. F. (Eds.). Historia Natural del Parque Nacional Cumbres de Monterrey, México. Edit. UANL, CONANP, Pronatura Noreste A. C., Consejo de Flora y Fauna Silvestre de Nuevo León, Conabio. México, 2013. 355 p.

Bezaury-Creel, J.; Gutiérrez C. D. Áreas naturales protegidas y desarrollo social en México. En: Capital natu- a ser más limitados o incluso inexistentes para el tema de la conservación. Además es importante mencionar que sólo Guadalajara cuenta con áreas naturales protegidas en el contexto urbano del área metropolitana, las que han sido promovidas por la sociedad civil local.

El análisis y contraste de ambos casos pone en evidencia la falta de coordinación entre los distintos órdenes de gobierno para lograr la gestión territorial de las ANP's, que hoy se convierten en espacios indispensables para lograr la calidad de vida de los habitantes de las ciudades. Asimismo, es indispensable considerar a las ANP's existentes como partes y componentes de un sistema que contribuyen de manera relevante al mantenimiento de múltiples servicios ambientales.

\section{Agradecimientos}

Esta investigación se llevó a cabo gracias al Programa de Apoyo a Proyectos de Investigación e Innovación Tecnológica (PAPIIT) de la Universidad Nacional Autónoma de México, Proyecto clave IA300516. ral de México Vol. II: Estado de conservación y tendencias de cambio. Edit. Conabio, México, pp. 385-431, 2009. Disponible en: http:/www.biodiversidad.gob.mx/pais/ pdf/CapNatMex/Vol\%20II/II09_Areas\%20naturales\%20 protegidas $\% 20 \mathrm{y} \% 20$ desarrollo\%20social $\% 20$ en $\% 20$ Mex. pdf consultado 23 de diciembre de 2015

Buizer, M.; Arts, B.; Kok, K. Governance, scale and the environment: the importance of recognizing knowledge claims in transdisciplinary arenas. Ecology and Society, 16(1), 2011. 
Burger, M. J.; Meijers, E. J. Form follows function? Linking morphological and functional polycentricity. Urban Studies, 49(5), 1127-1149, 2012.

Campos, G. L. Da Semarnat luz verde al Estadio de Rayados en Nuevo León ... con condiciones, Proceso, 6 de agosto, 2011. Consultado en enero de 2016. Disponible en: $<$ http:// www.proceso.com.mx/?p=278002>.

Cárdenas, M. Propietarios y ejidatarios contra OPD de la Primavera. Conciencia, Enero 18. 2014. Consultado en enero de 2016. Disponible en: <http://concienciapublica. com.mx/noticias-relevantes/20306/>.

Comberti, C.; Thornton, T. F.; Wylliede, E. V.; Patterson, T. Ecosystem services or services to ecosystems? Valuing cultivation and reciprocal relationships between humans and ecosystems. Global Environmental Change, 34, 247-262, 2015. doi: 10.1016/j.gloenvcha.2015.07.007

Conanp. Estrategia hacia 2040: una orientación para la conservación de las áreas naturales protegidas de México. Edit. Comisión Nacional de Áreas Naturales Protegidas, Secretaría de Medio Ambiente y Recursos Naturales, México, 2014.

De Castro, F.; Hogenboom, B.; Baud, M. Gobernanza ambiental en América Latina. Buenos Aires: Clacso, 2015.

De la Maza, E. J.; Cadena, G. R.; Piguerón, W. C. Estado actual de las áreas naturales protegidas de América Latina y El Caribe (Versión preliminar). Edit. Programa de las Naciones Unidas para el Medio Ambiente. Oficina Regional para América Latina y El Caribe, Quercus Consultoría Ecológica S.C. México, 2003.

De la Mora-De la Mora, G. Estrategias de conservación: la creación de áreas protegidas, Veredas. Revista del Pensamiento Sociológico, 5(9), 185-200, 2004.

De la Mora-De la Mora, G.; Montaño R. ¿Hacia la construcción de una gobernanza ambiental participativa, Estudio de caso en el Área Metropolitana de Guadalajara. Intersticios Sociales, Colegio de Jalisco, año 6, 11, 1-27, Marzo-Agosto 2016.

Douglas, H. A.; Dietz, T. Information, networks and the complexity of trust in commons governance. International Journal of the Commons, 5(2), 188-212, 2011.

Dudley, N.; Stolton, S. Running Pure: The Importance of Forest Protected Areas to Drinking Water. Edit. World Bank, WWF Alliance for Forest Conservation and Sustain- able Use, World Bank, 2003, Disponible en: <https://www. wdronline.worldbank.org/handle/10986/15006 License: CC BY 3.0 IGO>.

Fletcher, R. Neoliberal environmentality: Towards a poststructuralist political ecology of the conservation debate. Conservation and Society, 8(3), 171, 2010.

Foforocha, Gran Parque Ecológico La Pastora, 2010. Consultado en enero de 2016. Disponible en: $<$ https://www. youtube.com/watch? $\mathrm{v}=$ roMHLlBa45E $>$.

Gerez, P. Manejo forestal y protección de la biodiversidad: aprendizajes de dos políticas contradictorias. El Jarocho Verde. Red de Información y Acción Ambiental de Veracruz. (Riaave). México, Marzo 2001.

Gruby, R. L.; Basurto X. Multilevel governance for large marine commons: politics and polycentricity in Palau's protected area network. Environmental Science \& Policy, 260-272, 2013 doi: 10.106/j.envsci.2013.06.006

Heikkila, T.; Schlager, E.; Davis M. W. The role of crossscale institutional linkages in common pool resourcemanagement: assessing interstate river compacts. Policy Studies Journal, 39, 121-145, 2011.

Hesselbah, M. H.; Pérez C. M. S. Sistema de áreas naturales protegidas: estrategia para la conservación. Cuadernos de Trabajo. Agricultura y Recursos Naturales. Gobierno del Estado de Aguascalientes. México, Julio-Agosto, 1996.

Konijnendijk, van den B. C. C. From government to governance. Contribution to political ecology of urban forestry. En: Sandberg, L. A; Bardekjian, A.; Butt, S. Urban forests, trees and green space. A political ecology perspective. Edit. Earthscan-Routledge, 2015.

Merino, P. L. Conservación o deterioro. El impacto de las políticas públicas en las instituciones comunitarias y en los usos de los bosques de México. Edit. Semarnat, Ine, CCMSS. México, 2004.

Negrete, S. M. E. Las metropolis mexicanas: conceptualización, gestión y agenda política. En: Garza, G.; Schteingart, M. (Coord.). Los grandes problemas de México. TII. Desarrollo Urbano y regional. Edit. Colmex. México, 2010. p. 173-212.

Norgaard, R. B. Ecosystem services: from eye-opening metaphor to complexity blinder. Ecological Economics, 69, 2010. 
Ostrom, E. A general framework for analyzing sustainability of social-ecological systems. Science, 325, 550-557, 2009.

Ostrom, V.; Tiebout, C. M.; Warren, R. The organization of government in metropolitan areas: a theoretical inquiry. American Political Sciences Review, 55, 831-842, 1961.

Peters, G.; Pierre, J. Multi-level governance and democracy: a Faustian bargain? Multi-level Governance. En: Bache, I.; Flinders, M. (Eds.). Oxford, U.K.: Oxford University Press, 2004. p. 75-89.

Primack, R.; Rozzi, R.; Feinsigner, P.; Dirzo, R.; Massardo, F. Fundamentos de la conservación biológica. Perspectivas latinoamericanas. México: FCE, 2001.

Sartori, G. Concept misformation in comparative politics. American Political Science Review, 64(4), 1033-1053, 1970.

Simonian, L. La defensa de la tierra del Jaguar. Una historia de la conservación en México. Edit. Semarnap, Instituto de Ecología, Conabio, México, 1999. 345 p..

Sobrino, J. Zonas metropolitanas de México en 2000: conformación territorial y movilidad de la población ocu- pada (Parte A). Estudios demográficos y urbanos, México: Colmex, 54, 461-507, Sept.-Dic. 2003.

Soja, E. Entrevista a Edward Soja por Mariona Tomás. Café de las ciudades. Revista Digital, 22, 2004. Consultado en diciembre de 2015. Disponible en: $<$ http://www. cafedelasciudades.com.ar/politica_22.htm $>$ o $<$ http:// www.cafedelasciudades.com.ar/PDFs/cafe_de_las_ciudades_22_PDF.PDF>.

Stolton, S.; Dudley, N. Gracias a la ordenación forestal, la población urbana obtiene unos suministros hídricos más limpios. Unasylva: Revista Internacional de Silvicultura e Industrias Forestales, 229(58), 39-43, 2007. Disponible en: <ftp://ftp.fao.org/docrep/fao/010/a1598s/a1598s10.pdf>.

Van Meeteren, M.; Poorthuis, A.; Derudder, B.; Witlox, F. Pacifying Babel's Tower: a scientometric analysis of polycentricity in urban research. Urban Studies Journal, 2015. doi: $10.1177 / 0042098015573455$

Vasanen, A. Functional polycentricity: examining metropolitan spatial structure through the connectivity of urban sub-centres. Urban Studies, 49(16), 3627-3644, 2012. 